\title{
Wireless MIMO Switching with Zero-forcing Relaying
}

\author{
Fanggang Wang*†, Soung Chang Liew*, Dongning Guo ${ }^{\ddagger}$ \\ ${ }^{*}$ Institute of Network Coding, The Chinese Univ. of Hong Kong \\ ${ }^{\dagger}$ State Key Lab of Rail Traffic Control and Safety, EIE, Beijing Jiaotong Univ. \\ †Department of Electrical Engineering \& Computer Science, Northwestern Univ. \\ Email: fgwang@inc.cuhk.edu.hk, soung@ie.cuhk.edu.hk,dGuo@northwestern.edu
}

\begin{abstract}
A wireless relay with multiple antennas is called a multiple-input-multiple-output (MIMO) switch if it forms a one-to-one mapping from the inputs (uplinks) to the outputs (downlinks). This paper studies the case with $N$ source stations and $N$ destination stations (which may be the same set), so that the mapping is any permutation of the $N$ inputs. Moreover, the switching is achieved by "precode-and-forward", i.e., the relay precodes the received vector signal by a zero-forcing matrix and transmits it, so that each destination station receives only its desired signal with enhanced noise but no interference. Assuming full channel state information is available at the switch, the design of the zero-forcing precoder for maximizing some performance metric based on the received signal-to-noise ratios is investigated. The problem with two stations is completely solved in closed form in certain cases. In other cases, heuristic algorithms are proposed to optimize the precoder. These algorithms are shown to be nearoptimal.
\end{abstract}

Index Terms-Beamforming, fairness, maxmin, MIMO switching, relay, zero-forcing.

\section{INTRODUCTION}

Relays in wireless networks can extend coverage as well as improve energy efficiency [1]. In this paper, we study a set-up in which multiple stations communicate with each other via a multi-antenna relay. We let transmitters transmit simultaneously to the relay, then the relay precodes its received signal by a zero-forcing matrix before broadcasting to the receivers (which can be the same set as the transmitters). The precoder is such that a desired one-to-one mapping from the sources to the destinations is formed. Hence the relay is called a multiple-input multiple-output (MIMO) switch.

Prior work that investigated $N$ stations exchanging data via a relay includes $[1]-[5]$. References [2] $-[4]$ investigated the case of "full data exchange," in which all stations want to broadcast their data to all other stations. A slotted system is considered in [2] to optimize throughput, and the maximum throughput region is evaluated. However, it does not allow multiple packet reception at the relay. Data transmissions in [3], [4] can be summarized as follows: in the first slot, all

This work was partially supported by grants from the University Grants Committee of the Hong Kong Special Administrative Region, China (Project No. AoE/E-02/08; Project No. 414911); Program for Changjiang Scholars and Innovative Research Team in University (Grant No. IRT0949); the Joint Funds of State Key Program of NSFC (Grant No. 60830001); the Fundamental Research Funds for the Central Universities. stations transmit to the relay simultaneously; subsequent slots are devoted to downlink transmissions; in each downlink slot, the relay multiplies the signal received in the first time slot by a different precoder, such that at the end of all downlink slots, all stations receive the broadcast data from all other stations. By contrast, the framework investigated in this paper focuses on the pure unicast case, in which station $i$ transmits to another station $j$ only ${ }^{1}$ As indicated in our earlier paper [6], this unicast framework is more general in that it can realize any general transmission pattern (unicast, multicast, broadcast, or a mixture of them) among the stations by scheduling a set of different unicast transmissions.

Reference [1] studied both full data exchange and "pairwise data exchange," in which stations form pairs, and two stations in a pair exchange data with each other only. It is a special case of the unicast switching. In [1], a single-antenna relay with different forwarding strategies was considered in Gaussian channel. Reference [5] also studied pairwise data exchange, but the relay adopts the decode-and-forward strategy only. The diversity-multiplexing tradeoffs under reciprocal and nonreciprocal channels were analyzed. Both [1] and [5] studied the case of pairwise transmission. In this paper, we consider both pairwise and non-pairwise data exchanges among the stations, in which a multi-antenna relay works in precode-and-forward manner in Rayleigh fading channel.

Using our framework, we study switching traffic among the stations using a zero-forcing MIMO relay, where each destination receives the desired signal with enhanced noise. For fairness, we first restrict the precoder to yield identical received signal-to-noise ratio (SNR) by all stations and study how to design the precoder to maximize the throughput. We then lift the constraint and study how to design the precoder to maximize the minimum received SNR among all stations. The former problem is completely solved in the special case of two stations, assuming reciprocity between the uplink and the downlink channels. We also derive the conditions under which the two problems are equivalent. By evaluating the throughput performances of the two problems, we show the throughput gap between the two problems are trivial especially in the high

\footnotetext{
${ }^{1}$ Note that for general unicast transmission even if station $i$ transmits to station $j$, station $j$ does not necessarily transmits to station $i$ in the same transmission.
} 


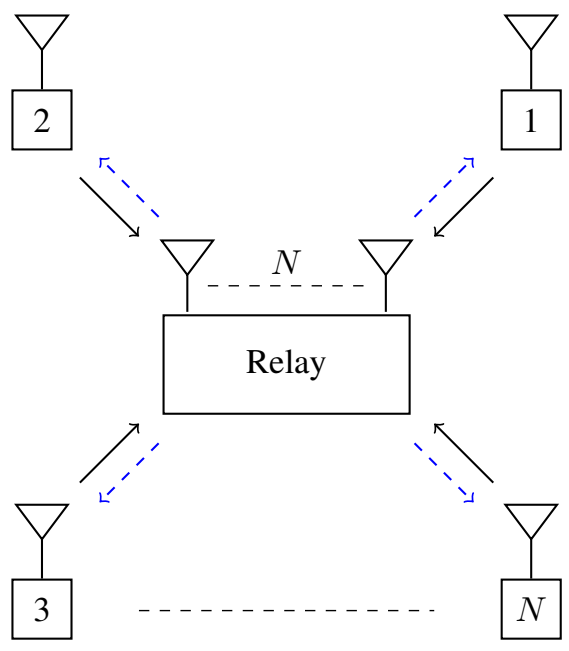

$\longrightarrow$ Uplink symbol interval

- - - -> Downlink symbol interval

Fig. 1. Wireless MIMO switching.

signal-to-noise ratio (SNR) regime. Lastly, we claim that we can use the former problem to approximate the latter problem: the latter being an NP-hard problem.

The remainder of the paper is organized as follows: Section II describes the framework of wireless MIMO switching and introduces the zero-forcing relaying method for establishing permutation among stations. An optimization problem requiring each station with equal post-processing SNR is studied in Section III. In Section IV, the problem of maximizing the minimum SNR of all users is investigated. Section V presents the simulation results. The paper is concluded by Section VI.

\section{SYSTEM DESCRIPTION}

Consider $N$ stations, numbered $1, \ldots, N$, each with one antenna, as shown in Fig. 1] The stations communicate via a relay with $N$ antennas and there is no direct link between any two stations. Each transmission comprises of one uplink symbol interval and one downlink symbol interval. The two symbol intervals could be two slots in a time-division system, or they could overlap in time when carried by different frequencies in a frequency-division system. The uplink symbol interval is for uplink transmissions from the stations to the relay; the downlink symbol interval is for downlink transmissions from the relay to the stations. We assume the two intervals are of equal duration. Each transmission realizes a switching permutation, as described below.

Consider one transmission. Let $\boldsymbol{x}=\left[x_{1}, \cdots, x_{N}\right]^{T}$ be the vector representing the signals transmitted by the stations. Let $\boldsymbol{y}=\left[y_{1}, \cdots, y_{N}\right]^{T}$ be the received signals at the relay, and $\boldsymbol{u}=\left[u_{1}, \cdots, u_{N}\right]^{T}$ be the noise vector with independent identically distributed (i.i.d.) noise samples following circularly-symmetric complex Gaussian (CSCG) distribution, i.e., $u_{n} \sim \mathcal{N}_{c}\left(0, \gamma^{2}\right)$. Then

$$
\boldsymbol{y}=\boldsymbol{H} \boldsymbol{x}+\boldsymbol{u},
$$

where $\boldsymbol{H}$ is the uplink channel gain matrix. The relay multiplies $\boldsymbol{y}$ by a precoding matrix $\boldsymbol{G}$ before relaying the signals. In this paper, we assume that the uplink channel and downlink channel are reciprocal, i.e., the downlink channel is $\boldsymbol{H}^{T}$. Thus, the received signals at the stations in vector form are

$$
\begin{aligned}
\boldsymbol{r} & =\boldsymbol{H}^{T} \boldsymbol{G} \boldsymbol{y}+\boldsymbol{w} \\
& =\boldsymbol{H}^{T} \boldsymbol{G} \boldsymbol{H} \boldsymbol{x}+\boldsymbol{H}^{T} \boldsymbol{G} \boldsymbol{u}+\boldsymbol{w}
\end{aligned}
$$

where $\boldsymbol{w}$ is the noise vector at the receiver, with the i.i.d. noise samples following CSCG distribution, i.e., $w_{n} \sim \mathcal{N}_{c}\left(0, \sigma^{2}\right)$.

We refer to an $N \times N$ matrix $\boldsymbol{P}$ that has one and only one nonzero element on each row and each column equal to 1 as a permutation matrix. Evidently, $\boldsymbol{P} \boldsymbol{x}$ is a column vector consisting of the same elements as $\boldsymbol{x}$ but permuted in a certain order depending on $\boldsymbol{P}$. For example, if

$$
\boldsymbol{P}=\left[\begin{array}{lll}
0 & 0 & 1 \\
1 & 0 & 0 \\
0 & 1 & 0
\end{array}\right]
$$

then

$$
\boldsymbol{P} \boldsymbol{x}=\left[x_{3}, x_{1}, x_{2}\right]^{T} .
$$

In the case where all diagonal elements of $\boldsymbol{P}$ are zero it is also called a derangement.

Suppose that the purpose of $\boldsymbol{G}$ is to realize a particular permutation represented by the permutation matrix $\boldsymbol{P}$, and to amplify the signals coming from the stations. That is,

$$
\boldsymbol{H}^{T} \boldsymbol{G} \boldsymbol{H}=\boldsymbol{A P},
$$

where $\boldsymbol{A}=\operatorname{diag}\left\{a_{1}, \cdots, a_{N}\right\}$ is an "amplification" diagonal matrix. Each diagonal element is regarded as the gain of a link. Accordingly, the precoder can be calculated by

$$
\boldsymbol{G}=\boldsymbol{H}^{-T} \boldsymbol{A} \boldsymbol{P} \boldsymbol{H}^{-1} .
$$

Let the receivers compensate for the amplification to yield received signal:

$$
\begin{aligned}
\hat{\boldsymbol{r}} & =\boldsymbol{A}^{-1} \boldsymbol{r} \\
& =\boldsymbol{P} \boldsymbol{x}+\boldsymbol{v},
\end{aligned}
$$

where the post-processing noise is expressed as

$$
\boldsymbol{v}=\boldsymbol{P} \boldsymbol{H}^{-1} \boldsymbol{u}+\boldsymbol{A}^{-1} \boldsymbol{w} .
$$

Let us define

$$
\Psi=\boldsymbol{I}+\gamma^{2} \boldsymbol{P} \boldsymbol{H}^{-1} \boldsymbol{H}^{-H} \boldsymbol{P}^{T} .
$$

The covariance of the post-processing noise $\boldsymbol{v}$ is written as

$$
\begin{aligned}
\mathbb{E}\left\{\boldsymbol{v} \boldsymbol{v}^{H}\right\} & =\gamma^{2} \boldsymbol{P} \boldsymbol{H}^{-1} \boldsymbol{H}^{-H} \boldsymbol{P}^{T}+\sigma^{2} \boldsymbol{A}^{-1} \boldsymbol{A}^{-H} \\
& =\Psi-\boldsymbol{I}+\sigma^{2} \boldsymbol{A}^{-1} \boldsymbol{A}^{-H} .
\end{aligned}
$$

Suppose all uplink transmissions use unit power, i.e.,

$$
\mathbb{E}\left\{x_{i}^{2}\right\}=1, \forall i \text {. }
$$


It is also fair to assume that the transmissions are independent, i.e., $\mathbb{E}\left\{x_{i} x_{j}^{*}\right\}=0, \forall i \neq j$. The problem is to design the precoder $G$ to minimize the variance of the post-processing noise subject to a power constraint on the signals transmitted by the relay:

$$
\mathbb{E}\left\{\boldsymbol{y}^{H} \boldsymbol{G}^{H} \boldsymbol{G} \boldsymbol{y}\right\} \leq q .
$$

For notational convenience, let us define an $N \times N$ matrix $\boldsymbol{S}$, whose entries are

$$
s_{i j}=\psi_{j i}\left[\left(\boldsymbol{H}^{*}\right)^{-1} \boldsymbol{H}^{-T}\right]_{i j} .
$$

The transmit power of the switch can be evaluated as

$$
\begin{aligned}
\mathbb{E} & {\left[\boldsymbol{x}^{H} \boldsymbol{H}^{H} \boldsymbol{G}^{H} \boldsymbol{G} \boldsymbol{H} \boldsymbol{x}+\boldsymbol{u}^{H} \boldsymbol{G}^{H} \boldsymbol{G u}\right] } \\
& =\operatorname{Tr}\left[\boldsymbol{G} \boldsymbol{H} \boldsymbol{H}^{H} \boldsymbol{G}^{H}\right]+\gamma^{2} \operatorname{Tr}\left[\boldsymbol{G} \boldsymbol{G}^{H}\right] \\
& =\operatorname{Tr}\left[\boldsymbol{H}^{-T} \boldsymbol{A}\left(\boldsymbol{I}+\gamma^{2} \boldsymbol{P} \boldsymbol{H}^{-1} \boldsymbol{H}^{-H} \boldsymbol{P}^{T}\right) \boldsymbol{A}^{H}\left(\boldsymbol{H}^{*}\right)^{-1}\right] \\
& =\operatorname{Tr}\left[\boldsymbol{A} \Psi \boldsymbol{A}^{H}\left(\boldsymbol{H}^{*}\right)^{-1} \boldsymbol{H}^{-T}\right] \\
& =\boldsymbol{a}^{H} \boldsymbol{S} \boldsymbol{a} .
\end{aligned}
$$

where $\boldsymbol{a}=\left[a_{1}, \cdots, a_{N}\right]^{T}$ is the gain vector with the diagonal elements of $\boldsymbol{A}$. The power constraint on the relay is thus expressed as

$$
\boldsymbol{a}^{H} \boldsymbol{S a} \leq q .
$$

We have thus established a framework for MIMO switching. Specifically, the permutation $\boldsymbol{P}$ is a switch matrix, which establishes a unicast pattern of transmission. We remark that the framework can be generalized to the case where the switch matrix does not realize a permutation, but a general transmission pattern. For example, if there are two nonzero elements in a column of $\boldsymbol{P}$, then a multicast connection is being realized within one switch matrix. We further remark that the permutation switch matrix framework nevertheless is rich enough to realize different traffic demands between the users. In particular, by scheduling a set of switch matrices, each realizing a permutation, we could satisfy general traffic flows among the users.

We provide a simple example to illustrate an application scenario of MIMO switching based on permutation switch matrices. Consider a network with three stations, 1, 2, and 3. The traffic flows among them are shown in Fig. 2 2 station 1 wants to transmit " $a$ " to both stations 2 and 3 ; station 2 wants to transmit " $b$ " and " $c$ " to stations 1 and 3 , respectively; station 3 wants to transmit " $d$ " and " $e$ " to stations 1 and 2 , respectively. Pairwise data exchange as in [1] and [5] is not effective in this case because when the number of stations is odd, one station will always be left out when forming pairs. That is, when the number of stations is odd, the connectivity pattern realized by a switch/permutation matrix does not correspond to pairwise communication. Full data exchange is not appropriate either, since in our example, station 2 (as well as station 3) transmits different data to the other two stations. Under our framework, the traffic flows among stations can be met as shown in Fig. 3. In the first transmission, station 1 transmits " $a$ " to station 3 ; station 2 transmits " $b$ " to station 1 ; station 3 transmits " $e$ " to station 2 . In the second transmission,
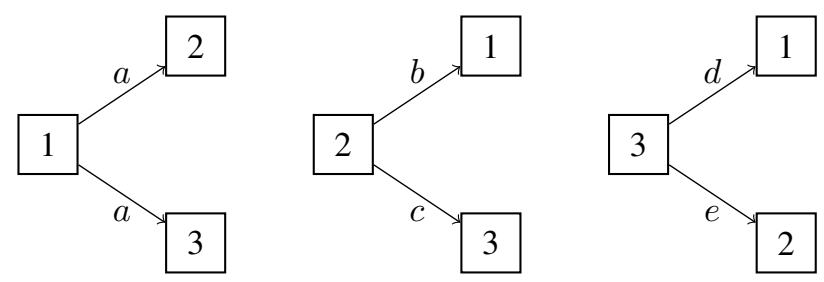

Fig. 2. Traffic demand of a three stations example.

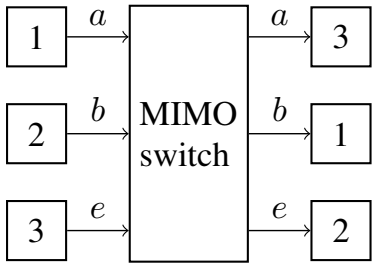

Transmission 1

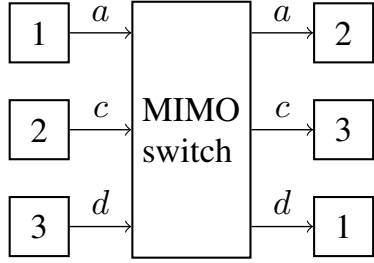

Transmission 2
Fig. 3. A MIMO switching established by two transmissions realizes the traffic demand in Fig. 2

station 1 transmits " $a$ " to station 2 ; station 2 transmits " $c$ " to station 3 ; station 3 transmits " $d$ " to station 1 .

In this paper, we will present the details on how to design precoders to maximize the throughput under a fairness requirement. To limit the scope, this paper focuses on the use of zero forcing relaying to establish the permutation among stations. In general, the MIMO switching system can realize any general transmission pattern (unicast, multicast, broadcast, or a mixture of them) among the stations by scheduling a set of permutation switch matrices [6].

\section{The EQUAL-SNR PROBleM}

In this section, we focus on the problem of guaranteeing the maximum equal received SNR at all stations, called the equalSNR problem, which was originally proposed in [6]. According to $(8)$, the post-processing noise power of each station is $\epsilon=$ $\psi_{i}-1+\frac{\sigma^{2}}{\left|a_{i}\right|^{2}}$. Thus, the received SNR is $\frac{1}{\epsilon}$. Since the system is half-duplex with uplink and downlink of equal duration, the throughput achieved by Gaussian signaling is

$$
c_{i}=\frac{1}{2} \log _{2}\left(1+\frac{1}{\epsilon}\right),
$$

where the unit of $c_{i}$ is bits per symbol period.

\section{A. Problem Formulation}

Let $\boldsymbol{a}=\left[a_{1}, \cdots, a_{N}\right]^{T}$ be the link gains, i.e., the diagonal of the amplification matrix $\boldsymbol{A}$. An optimization problem is formulated as follows:

$$
\min _{\boldsymbol{a}} \epsilon
$$

subject to $\quad\left|a_{i}\right|^{2}=\frac{\sigma^{2}}{\epsilon+1-\psi_{i}}, 1 \leq i \leq N$,

$$
\boldsymbol{a}^{H} \boldsymbol{S a} \leq q,
$$

$$
\epsilon \geq 0 \text {, }
$$


where $\psi_{i} \triangleq \Psi_{i i}$. In [6], we only provided suboptimal solutions. In this paper, we will investigate optimal solutions and propose analytical and numerical suboptimal solutions for MIMO switching.

Lemma 1: Every optimal solution for the optimization problem (15) must satisfy the relay power constraint 15c with equality.

Proof: Let $\hat{\boldsymbol{a}}=\left[\hat{a}_{1}, \cdots, \hat{a}_{N}\right]^{T}$ be the optimal solution for the problem (15) with the optimal objective $\hat{\epsilon}$. Suppose $\hat{\boldsymbol{a}} \boldsymbol{S} \hat{\boldsymbol{a}}<q$. So there exists a positive number $\tau$, for all $\left|a_{i}\right|^{2} \in$ $\left(\left|\hat{a}_{i}\right|^{2}-\tau,\left|\hat{a}_{i}\right|^{2}+\tau\right), 1 \leq i \leq N$,

$$
\boldsymbol{a}^{H} \boldsymbol{S a}<q .
$$

Choose any number $\xi$ satisfying

$$
0<\xi \leq \min _{i} \hat{\epsilon}+1-\psi_{i}-\frac{1}{\frac{\tau}{2 \sigma^{2}}+\frac{1}{\hat{\epsilon}+1-\psi_{i}}},
$$

such that

$$
\max _{i} \frac{\sigma^{2}}{\hat{\epsilon}+1-\xi-\psi_{i}}-\frac{\sigma^{2}}{\hat{\epsilon}+1-\psi_{i}} \leq \frac{\tau}{2} .
$$

Let

$$
\left|\hat{a}_{i}^{\prime}\right|^{2}=\frac{\sigma^{2}}{\frac{\sigma^{2}}{\left|\hat{a}_{i}\right|^{2}}-\xi}, 1 \leq i \leq N .
$$

Then

$$
\max _{i}\left|\hat{a}_{i}^{\prime}\right|^{2}-\left|\hat{a}_{i}\right|^{2} \leq \frac{\tau}{2}, 1 \leq i \leq N .
$$

Thus, the power constraint holds with $\hat{\boldsymbol{a}}^{\prime}=\left[\hat{a}_{1}^{\prime}, \cdots, \hat{a}_{N}^{\prime}\right]^{T}$. In addition, it can be easily proved that the equal noise power constraint holds with $\hat{\boldsymbol{a}}^{\prime}$ by plugging 19 into this constraint, i.e., all the noise powers are the same,

$$
\psi_{i}-1+\frac{\sigma^{2}}{\left|\hat{a}_{i}\right|^{2}}-\xi=\hat{\epsilon}-\xi, 1 \leq i \leq N .
$$

However the noise power is $\hat{\epsilon}-\xi$ with $\hat{\boldsymbol{a}}^{\prime}$, smaller than the optimal objective $\hat{\epsilon}$, which proves that $\hat{\boldsymbol{a}}$ is not the optimal solution. This contradicts the assumption.

Lemma 1 is a key property to solve the optimization problems. With the property, we could eliminate part of the feasible solutions, i.e., the solutions with which the relay consumes less power than $q$.

Proposition 1: The equal-SNR problem is feasible, i.e., its feasible solutions always exist.

The proof can be found in [6]. In the following, we explore the optimal or suboptimal solution of $(15)$.

\section{B. Optimal Solution in the Case of $N=2$}

We consider the case of two stations. The power constraint of the relay in 15 can be expanded as

$$
\boldsymbol{a}^{H} \boldsymbol{S} \boldsymbol{a}=s_{11}\left|a_{1}\right|^{2}+s_{22}\left|a_{2}\right|^{2}+s_{21} a_{1} a_{2}^{*}+s_{12} a_{1}^{*} a_{2},
$$

where $s_{i j}$ is element $(i, j)$ of $\boldsymbol{S} ; s_{11} \geq 0, s_{22} \geq 0$, and $s_{12}=s_{21}^{*}$. By reciprocity of the uplink and downlink channels, $s_{12}=s_{21} \in \mathbb{R}^{+}$. The formula 22 can be written as

$$
\boldsymbol{a}^{H} \boldsymbol{S} \boldsymbol{a}=\left|\sqrt{s_{11}} a_{1}+\frac{s_{12}}{\sqrt{s_{11}}} a_{2}\right|^{2}+\left(s_{22}-\frac{s_{12}^{2}}{s_{11}}\right)\left|a_{2}\right|^{2} .
$$

Since the term $\boldsymbol{a}^{H} \boldsymbol{S} \boldsymbol{a}$ is quadratic, the trace of the matrix is always positive, then we have $s_{11} s_{22} \geq s_{12}^{2}$.

In order to minimize the post-processing noise power $\epsilon$, we should try to maximize $\left|a_{1}\right|$ and $\left|a_{2}\right|$. Given a value of $\epsilon$, the amplitudes $\left|a_{1}\right|$ and $\left|a_{2}\right|$ can be calculated by the equal noise power constraint. Then we should explores the optimal phases to minimize the relay power consumption. We see that only $\left|\sqrt{s_{11}} a_{1}+\frac{s_{12}}{\sqrt{s_{11}}} a_{2}\right|^{2}$ is related to the phases of variables $a_{1}$ and $a_{2}$ in (23). It is easy to see the global minimum is achieved by real-valued $a_{1}$ and $a_{2}$ with opposite signs. Without loss of optimality, assume $a_{1} \in \mathbb{R}^{+}$and $a_{2} \in \mathbb{R}^{-}$. Then the power consumption constraint can be simplified by

$$
\begin{aligned}
& \left(\sqrt{s_{11}} a_{1}+\frac{s_{12}}{\sqrt{s_{11}}} a_{2}\right)^{2}+\left(s_{22}-\frac{s_{12}^{2}}{s_{11}}\right) a_{2}^{2} \\
& =s_{11} a_{1}^{2}+2 s_{12} a_{1} a_{2}+s_{22} a_{2}^{2}=q .
\end{aligned}
$$

According to the equal noise power constraint, we have

$$
a_{2}=-\frac{\sigma}{\sqrt{\psi_{1}-\psi_{2}+\frac{\sigma^{2}}{a_{1}^{2}}}} .
$$

Plugging (25) into the power constraint, we have (26).

$$
\begin{aligned}
& s_{11}^{2} \psi_{\delta}^{2} a_{1}^{8}+2 \psi_{\delta}\left[s_{11}^{2} \sigma^{2}+s_{11} s_{22} \sigma^{2}-\right. \\
& \left.s_{11} q \psi_{\delta}-2 s_{12}^{2} \sigma^{2}\right] a_{1}^{6}+\left[\sigma^{4}\left(s_{11}+s_{22}\right)^{2}+\right. \\
& \left.q^{2} \psi_{\delta}^{2}-2 q \sigma^{2} \psi_{\delta}\left(2 s_{11}+s_{22}\right)-4 s_{12}^{2} \sigma^{4}\right] a_{1}^{4}+ \\
& {\left[2 q^{2} \sigma^{2} \psi_{\delta}-2 q \sigma^{4}\left(s_{11}+s_{22}\right) a_{1}^{2}+q^{2} \sigma^{4}=0,\right.}
\end{aligned}
$$

where $\psi_{\delta}=\psi_{1}-\psi_{2}$. Since the equal-SNR problem is feasible according to Proposition 11, thus, there exists solutions for (26). In order to maximize $a_{1}$ and $a_{2}$, the largest real root of the corresponding equation of 26) is the optimal solution of $a_{1}$. Since this equation is biquartic, it has an analytical solution, which can be found in [7]. With the analytical solution we can solve for $a_{1}$, and then $a_{2}$ is calculated by 25 .

Alternatively, after we deduce that $a_{1}$ and $a_{2}$ have opposite phases, a numerical method can be used to solve the problem as well, which will be introduced in the following subsection. Thus, we have solved the equal-SNR problem of 15$)$ in the case of $N=2$.

It is not difficult to see that making the two user signals having opposite phases minimizes the relay power consumption. As we shall see, in case of more than two users with users forming pairs, assigning each pair of users opposite phases is an effective solution. Thus, in general the counter-phase setting is effective for pairwise transmission in any problem which needs to minimize the relay power consumption, including the maxmin problem introduced in the following section.

We have solved the equal-SNR problem when $N=2$, but do not have any effective method to find the optimal solutions for larger $N$ at this moment. For practical implementation with quick computation time, we propose suboptimal schemes as follows. 
C. Suboptimal Solution with Real Positive Gains in the Case of $N>2$

We consider a general case of either pairwise or nonpairwise transmission for any $N$. First, we restrict the elements of $\boldsymbol{a}$ to be non-negative real numbers. A numerical method is provided to find the real-valued solution. The phases will be optimized in the next subsection.

Recall that the equal noise power constraint in (15b), then we have

$$
a_{j}=\frac{\sigma}{\sqrt{\epsilon+1-\psi_{i}}}, \quad \forall j
$$

According to Lemma 1, we use "=" instead of " $\leq$ " in the power consumption constraint in $15 \mathrm{c}$, , i.e.,

$$
\boldsymbol{a}^{H} \boldsymbol{S a}=q,
$$

We now consider the problem of finding the real-valued solution to 15 .

Problem Definition: Given $\boldsymbol{H}, q, \sigma^{2}, \gamma^{2}$, and a desired permutation $\boldsymbol{P}$, solve for $\boldsymbol{G}$ and $\epsilon$.

There are $N$ equations in (27) and one equation in (28). These equations can be used to solve $a_{j} \forall j$ and $\epsilon$. After that (4) can be used to find $G$ from $\boldsymbol{a}$. An effective numerical method is proposed as follows.

Numerical Method $\Pi$ : We can solve for $\epsilon$ by one-dimensional search, which starts from $\max _{j} \psi_{j}-1$, i.e., when the power consumption of the relay approaches infinity. In each step of the search, $\epsilon$ is increased by a small number $\delta$. Given an $\epsilon$, we can calculate all $a_{j}$ 's according to the equal noise power constraint. After that, we check if the the power constraint holds with the temporary solution. Note that when the step size $\delta$ is small enough, the first feasible solution when we increase $\epsilon$ from $\max \psi_{j}$ is the optimal solution with the minimum $\epsilon$, then we do not need to continue searching for other feasible solutions.

D. Suboptimal Solution with Complex-valued Gains in the Case of $N>2$

We now consider the general case of complex-valued $\boldsymbol{a}$. With numerical method $\Pi$, the optimization of $a_{j}$ 's amplitudes and phases can be decoupled. According to Proposition 1 . there exists an $\epsilon$ such that $\boldsymbol{a}^{H} \boldsymbol{S a}=q$. Denote such an $\epsilon$ by $\epsilon\left(\theta_{1}, \cdots, \theta_{N}\right)$ since in each step we regard the amplitudes as constant values, where $\theta_{j}=\angle a_{j}$. The problem consists of finding

$$
\epsilon^{*}=\arg \min _{\theta_{1}, \cdots, \theta_{N}} \epsilon\left(\theta_{1}, \cdots, \theta_{N}\right) .
$$
15.).

In the following we investigate how to solve the problem

Random-phase Algorithm: In (29), we note that $\epsilon$ is a complicated nonlinear function of $\theta_{j}$. A time-consuming exhaustive search can be used to find the solution to (29). Instead, we use a random-phase algorithm to reduce the complexity. We divide the interval of $[0,2 \pi)$ equally into $M$ bins with the values of $0, \frac{2 \pi}{M}, \cdots, \frac{2(M-1) \pi}{M}$ respectively, and we randomly pick among them to set the the value of $\theta_{j}$ for each and every $j=1, \cdots, N$. After that, we compute the corresponding $\epsilon\left(\theta_{1}, \cdots, \theta_{N}\right)$ by solving $15 \mathrm{~b}$ ) and 28 . We perform $L$ trials of these random phase assignments to obtain $L$ feasible solutions. We choose the one using the least energy as our estimate for $\epsilon^{*}$. Substituting the estimated $\epsilon^{*}$ into 27) yields $\left|a_{j}\right|$ for all $j$; hence $G$. This best-out-of- $L$ feasible solution is in general larger than the actual optimal $\epsilon^{*}$. In Section VI, we will show that large gains can be achieved with only small $M$ and $L$. Moreover, increasing $M$ and $L$ further yields very little improvement, suggesting that the estimated $\epsilon^{*}$ with small $M$ and $L$ is perhaps near optimal.

Counter-phase Algorithm: Recall that in case of $N=2$ the solution has $a_{1}$ and $a_{2}$ with opposite signs, or in general the minimum is achieved with $a_{1}$ and $a_{2}$ being complex numbers with opposite phases, i.e., $\theta_{1}=\theta_{2}+\pi$. For large even number of $N$, consider the situation in which the transmissions are pairwise. The stations form pairs, and two stations in a pair exchange data with each other only. We could set a pair of two stations with counter phases. Our simulation results indicate that when the relative phase is $\pi$ within a pair, we can get roughly the same throughput performance by varying the phases of different pairs. Thus, to simplify the problem we use real numbers for the elements of $\boldsymbol{a}$. The amplitudes are calculated in the same way as that of the random-phase algorithm. The only difference between the two algorithms is how to set the phases.

\section{MAXMin PROBLEM}

In this section, we formulate another optimization problem, i.e., the maxmin problem, in which the minimum received SNR among all the stations is maximized. Since the requirement of the received SNR is relaxed, the throughput performance should be better than the equal-SNR problem.

The problem is formulated as

$$
\begin{array}{cl}
\min _{\boldsymbol{a}} & \epsilon \\
\text { subject to } & \left|a_{i}\right|^{2} \geq \frac{\sigma^{2}}{\epsilon+1-\psi_{i}}, 1 \leq i \leq N, \\
& \boldsymbol{a}^{H} \boldsymbol{S a} \leq q, \\
& \epsilon \geq 0 .
\end{array}
$$

The only difference between the formulations of the maxmin problem and the equal-SNR problem is $30 \mathrm{~b}$, i.e., we use "inequality" instead of "equality". First we prove a lemma of the maxmin problem $30{ }^{2}$, similar to Lemma 1.

Lemma 2: Every optimal solution for the optimization problem 30 must satisfy the relay power constraint 30c with equality.

Proof: We assume $\tilde{\boldsymbol{a}}=\left[\tilde{a}_{1}, \cdots, \tilde{a}_{N}\right]^{T}$ is the optimal solution for the problem 30 with the optimal objective $\tilde{\epsilon}$.

\footnotetext{
${ }^{2}$ Essentially, it is a minmax problem with respect to (w.r.t.) the postprocessing noise power. In this paper, we call it the maxmin problem w.r.t. the received SNR to keep it consistent with the following equal-SNR problem proposed in Section IV.
} 
Suppose $\tilde{\boldsymbol{a}} \boldsymbol{S} \tilde{\boldsymbol{a}}<q$. So there exists a positive number $\tau$, for all $\left|a_{i}\right| \in\left(\left|\tilde{a}_{i}\right|-\tau,\left|\tilde{a}_{i}\right|+\tau\right), 1 \leq i \leq N$,

$$
\boldsymbol{a}^{H} \boldsymbol{S a}<q \text {. }
$$

Let

$$
\left|\tilde{a}_{i}^{\prime}\right|=\left|\tilde{a}_{i}\right|+\frac{\tau}{2}, 1 \leq i \leq N
$$

and let

$$
\Xi_{i}\left(a_{i}\right) \triangleq \psi_{i}+\frac{\sigma^{2}}{\left|a_{i}\right|^{2}}
$$

Then

$$
\Xi_{i}\left(\tilde{a}_{i}^{\prime}\right)<\Xi_{i}\left(\tilde{a}_{i}\right), 1 \leq i \leq N .
$$

Since each $\Xi_{i}\left(\tilde{a}_{i}^{\prime}\right)$ is smaller than $\Xi_{i}\left(\tilde{a}_{i}\right)$ for all $i$, the minmax objective becomes smaller with the solution $\tilde{\boldsymbol{a}}^{\prime}=\left[a_{1}^{\prime}, a_{2}^{\prime}\right]^{T}$. Thus, $\tilde{\boldsymbol{a}}$ is not the optimal solution, which contradicts the assumption.

Similarly, we could eliminate the feasible solutions with which the relay consumes less power than $q$ based on Lemma 2. Note that the optimal solution of the maxmin problem when $N=2$ also has the opposite-sign property.

Another property is that the optimal objective of the equalSNR problem (15) is the upper bound of the optimal objective of the maxmin problem (30). The solution of the equalSNR problem is feasible for the maxmin problem, since the constraint of the maxmin problem is one of the constraint in the equal-SNR problem. Thus, the optimal objective of (15) can not be smaller than that of (30), otherwise the optimal solution to $(15)$ is a better solution to $(30)$ than its original optimal solution.

However, the maxmin problem is NP-hard, which is indicated by the following proposition.

Proposition 2: The maxmin problem 30] is NP-hard in the size of $N$ when $\gamma \neq 0$.

Proof: First we will find an equivalent problem of 30 . Then we prove the equivalent problem is NP-hard.

Let $\tilde{\boldsymbol{a}}$ be an optimal solution of $(30)$, and the associated optimal objective is $\tilde{\epsilon}$. Note that $\tilde{\epsilon}$ is the maximum noise power for all $i$. Then we formulate another optimization problem as below:

$$
\begin{array}{cl}
\min _{\boldsymbol{a}} & \boldsymbol{a}^{H} \boldsymbol{S a} \\
\text { subject to } & \left|a_{i}\right|^{2} \geq \frac{\sigma^{2}}{\tilde{\epsilon}+1-\psi_{i}}, 1 \leq i \leq N, \\
& \tilde{\epsilon} \geq 0 .
\end{array}
$$

The solution $\tilde{\boldsymbol{a}}$ satisfies the constraints of $[35$, where the largest noise power among all $i$ is equal to $\tilde{\epsilon}$. Hence, $\tilde{\boldsymbol{a}}$ is a feasible solution of (35). According to Lemma 2, the power consumption of the relay is $\tilde{\boldsymbol{a}}^{H} \boldsymbol{S} \tilde{\boldsymbol{a}}=q$. Let $\hat{\boldsymbol{a}}$ be an optimal solution of (35). The power consumption of the relay can not be larger than $q$, otherwise $\hat{\boldsymbol{a}}$ is even worse than $\tilde{\boldsymbol{a}}$ for 35 . If the power consumption of the relay $\hat{\boldsymbol{a}}^{H} \boldsymbol{S} \hat{\boldsymbol{a}}$ is strictly smaller than $q$, then $\hat{\boldsymbol{a}}$ is a feasible solution of 30 , which is at least as good as $\tilde{\boldsymbol{a}}$ since the maximum noise power is not larger
TABLE I

THROUGHPUTS OF THE EQUAL-SNR PROBLEM (COUNTER-PHASE ALGORITHM) AND THE MAXMIN PROBLEM (EXHAUSTIVE SEARCH) WHEN $N=2 ; \Delta$ DENOTES THE VARIATIONAL RATIO WITH RESPECT TO (W.R.T.) THE EQUAL-SNR SOLUTION OF THE COUNTER-PHASE ALGORITHM.

\begin{tabular}{cccc}
\hline SNR(dB) & Equal-SNR & Maxmin(wst. $) / \Delta(\%)$ & Maxmin(avg. $) / \Delta(\%)$ \\
\hline 0 & 0.1270 & $0.1271 / 0.08$ & $0.1271 / 0.08$ \\
10 & 0.7990 & $0.7993 / 0.04$ & $0.7994 / 0.05$ \\
20 & 2.1249 & $2.1254 / 0.02$ & $2.1256 / 0.03$ \\
30 & 3.7075 & $3.7081 / 0.016$ & $3.7083 / 0.02$ \\
\hline
\end{tabular}

than $\tilde{\epsilon}$. That is, $\hat{\boldsymbol{a}}$ is an optimal solution of 30 . However, the power consumption $\hat{\boldsymbol{a}}^{H} \boldsymbol{S} \hat{\boldsymbol{a}}<q$ contradicts Lemma 2. Thus, $\hat{\boldsymbol{a}}^{H} \boldsymbol{S} \hat{\boldsymbol{a}}=q$. Furthermore, there is at least one constraint in (35) in which equality holds for $\hat{\boldsymbol{a}}$, otherwise $\hat{\boldsymbol{a}}$ is a better solution for (30). We have proved the optimal solution of (30) is also the optimal solution of (35), vice versa. Therefore, the two problems are equivalent.

The problem [35] is equivalent to the problem (2) in [8], which has been proved NP-hard in general. We do not repeat the steps in [8].

Proposition 3: If no additional noise is introduced at the switch, then the optimal solution of 30 is such that each station has exactly the same post-processing noise power. That is, in this case, the two optimization problems 30 and 15 are equivalent.

Proof: As proved in Proposition 2, the optimization problem (35) is equivalent to 30. When $\gamma=0$ in 35, $\psi_{i}=1 \forall i$. The objective can be rewritten as $\sum_{i} s_{i i}\left|a_{i}\right|^{2}$. The optimal solution of $\boldsymbol{a}$ is obvious, and it satisfies $\left|a_{i}\right|^{2}=\frac{\sigma^{2}}{\tilde{\epsilon}}, \forall i$. That is, for the optimal solution of [30], each station has equal post-processing noise power $\tilde{\epsilon}$.

Note that the maxmin problem can also guarantee fairness, but not perfect fairness. The post-processing noise powers may vary among all the stations. Regarding the fairness issue, the performance is mostly bottlenecked by the worst station. In Section VI, we will evaluate both the equal-SNR problem and the maxmin problem to show there is only a small performance gap between one station in the equal-SNR problem and the worst station in the maxmin problem. In addition, with the equal-SNR setting, MIMO switching can be easily extended to multiple transmissions, by which general transmission pattern can be realized [6]. However, if multiple transmissions with the maxmin setting are scheduled together, the performance of each station may vary even more widely, which goes against the fairness requirement. Moreover, the maxmin problem is NP-hard, whereas requires exhaustive search, while the equalSNR problem is practical for implementation. For the above reasons, we believe the equal-SNR setting is perhaps more amenable to practical deployment.

\section{Numerical Results}

In this section, we evaluate the throughputs of different schemes. We assume the maximum transmit power of the relay is the same as that of the stations, i.e., $q=1$, and the noise powers at the relay and the stations are the same. Our 


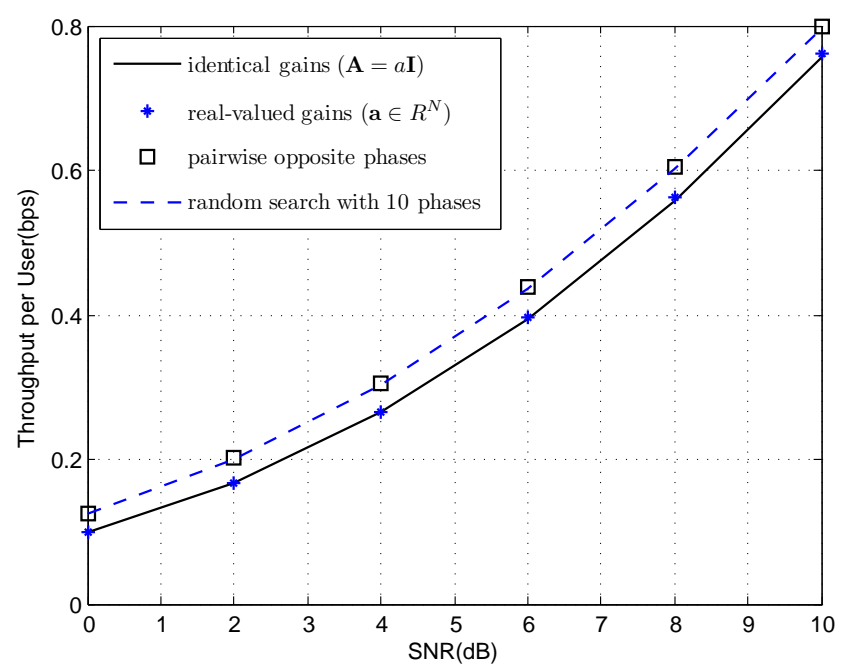

Fig. 4. Throughput comparison of different equal-SNR precoding schemes in the case of $N=2$.

simulation indicates that the system throughputs are roughly the same with different symmetric permutations. The same result can be concluded for asymmetric permutations. Thus, we just pick one permutation for each of them $\left(\boldsymbol{P}^{s}\right.$ and $\boldsymbol{P}^{a}$ as above) when presenting our results here.

$$
\boldsymbol{P}^{s}=\left[\begin{array}{llll}
0 & 0 & 0 & 1 \\
0 & 0 & 1 & 0 \\
0 & 1 & 0 & 0 \\
1 & 0 & 0 & 0
\end{array}\right], \boldsymbol{P}^{a}=\left[\begin{array}{llll}
0 & 0 & 1 & 0 \\
1 & 0 & 0 & 0 \\
0 & 0 & 0 & 1 \\
0 & 1 & 0 & 0
\end{array}\right] .
$$

We sum up our observations under three general results, as stated below.

General Result 1: The average throughput of the maxmin problem is larger than that of the equal-SNR problem. However, the throughput performance of the maxmin problem is mostly limited by the worst station. The throughput gap between one station in the equal-SNR problem and the worst station in the maxmin problem is small over a wide range of SNR.

We evaluate the throughput performances when $N=2$. An interesting question is how large is the throughput gap between (30) and (15) corresponding to their optimal noise powers. In Table I, the gap is evaluated over a wide range of SNR. Note that the optimal solutions of the maxmin problem are found by exhaustive search. The simulation results indicate that the throughputs of the maxmin problem is always larger than that of the equal-SNR problem, but the gap decreases as SNR becomes higher. In addition, the gap is less than $0.1 \%$ even for SNR as low as $0 \mathrm{~dB}$. Therefore, the maxmin problem can be well approximated by the equal-SNR problem.

Although the average throughput gap is larger, it is still less than $0.1 \%$ even for the SNR of $0 \mathrm{~dB}$, as indicated in Table I. Since we focus on the fairness issue, the former gap is the one of interest.

We note that the trend as indicated by the simulations results in Table I is consistent with the analytical result of Proposition

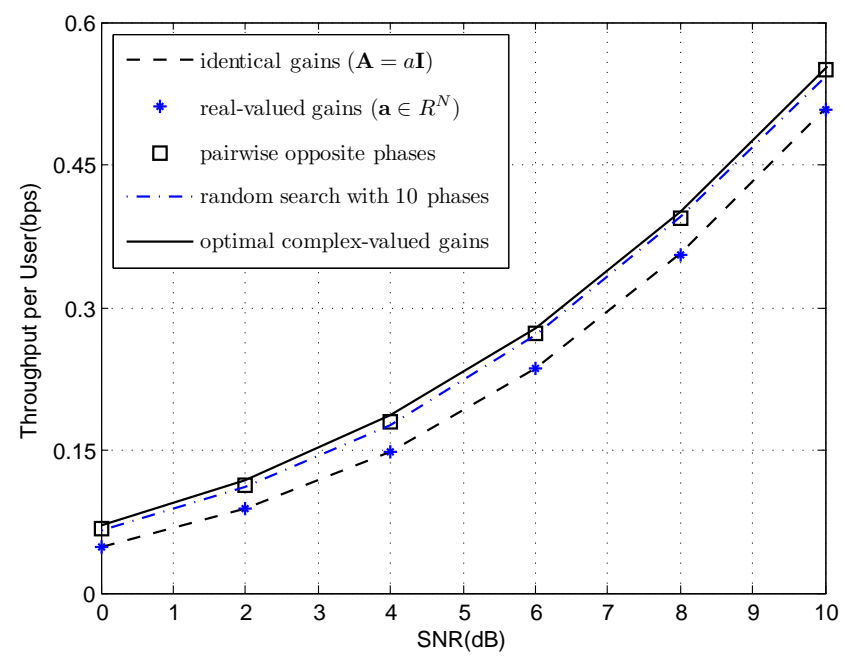

Fig. 5. Throughput comparison of different equal-SNR precoding schemes for pairwise switching pattern in the case of $N=4$.

3 In the high SNR regime, the noise becomes negligible, and the gap between equal-SNR and maxmin approaches to zero.

General Result 2: The optimal setting for $N=2$ has the property that the two elements of $\boldsymbol{a}$ have opposite signs. In general, the counter-phase algorithm is an effective scheme for pairwise transmission in the case of large even $N$.

As shown in Fig. 4, the scheme in $[9]^{3}$ has roughly the same average throughput as our MIMO switching scheme with realvalued $a$. Henceforth we regard the MIMO switching scheme with real-valued $\boldsymbol{a}$ as a benchmark and call it "the basic scheme". Despite having roughly the same average throughput performance, the basic scheme has an advantage over the scalar scheme in [9] in that the basic scheme guarantees fairness. That is, in our basic scheme, each station has exactly the same throughput, while the stations in the scheme in [9] may have different throughputs. Note that all the schemes proposed in this paper to solve the equal-SNR problem guarantee perfect fairness as the basic scheme does.

Compared with the basic scheme, the optimal setting of $N=2$, i.e., the counter-phase algorithm proposed in Section III.D, achieves more than $0.6 \mathrm{~dB}$ gain in low SNR regime. The gain becomes smaller as the SNR increases, e.g., around $0.4 \mathrm{~dB}$ gain at the SNR of $10 \mathrm{~dB}$. We explain why the gain becomes trivial for high SNR as follows. When the relay noise power is zero, $\left|a_{1}\right|=\left|a_{2}\right|$. In this case, $s_{12}$ becomes 0 in 23). Then the throughput performance does not depend on the phase difference of $a_{1}$ and $a_{2}$. The opposite-sign setting is equivalent to the identical-gain setting, i.e., the scheme in [9]. Thus, the gain over the basic scheme becomes trivial in the high-SNR regime.

In Fig. 5 when $N=4$, the four stations form two pairs for pairwise transmission. Our simulation results indicate that

\footnotetext{
${ }^{3} \mathrm{~A}$ similar framework as ours is investigated in [9], which focuses on optimizing the sum rate of all stations. Therein, a suboptimal beamforming scheme is proposed, which simply uses a positive scalar weight to control the relay power consumption instead of our diagonal $\boldsymbol{A}$.
} 


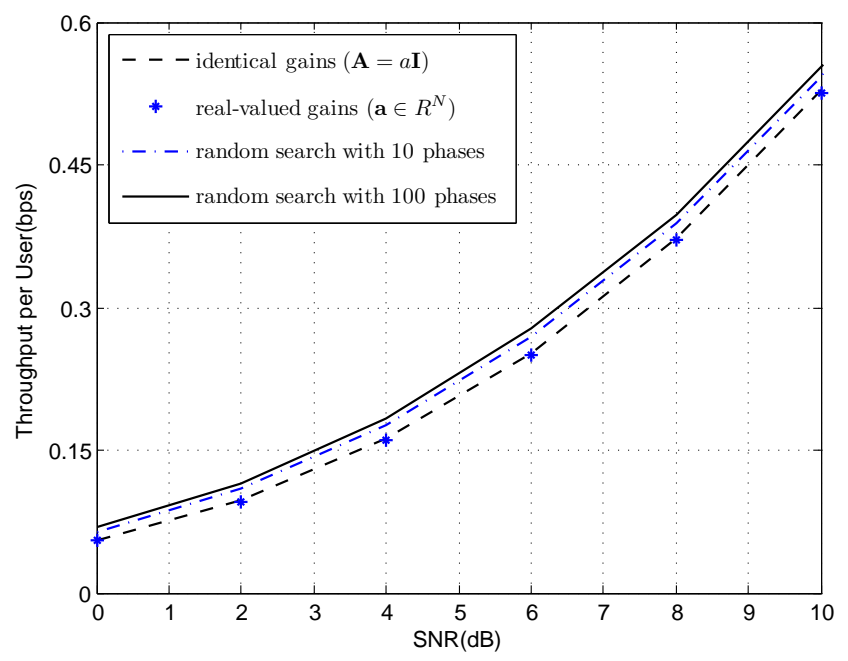

Fig. 6. Throughput comparison of different equal-SNR precoding schemes for non-pairwise switching pattern in the case of $N=4$.

the throughputs are roughly the same when we vary the phase differences of different pairs while keeping the relative phase difference within one pair to be $\pi$. These results are not shown in Fig. 5 to avoid cluttering. With this result, we could set the elements of $\boldsymbol{a}$ such that one element in each pair is positive and the other element is negative. The counter-phase scheme achieves $0.8 \mathrm{~dB}$ gain in the low SNR regime and $0.45 \mathrm{~dB}$ gain in the high SNR regime.

General Result 3: For the random-phase algorithms, simulation results indicate that large gains can be achieved with only small $M$ and $L$. It helps not only for the pairwise switching pattern but also for the non-pairwise pattern.

In Fig. 4 and Fig. 5, when $M=8$ and $L=10$, the random-phase scheme can achieve near optimal throughput performance. Consider the non-pairwise transmission in Fig. 6 . Note that the counter-phase scheme can not be applied to non-pairwise transmissions. The throughputs of the scalar scheme [9] and the real-valued $\boldsymbol{a}$ scheme are roughly the same. However, the random-phase scheme can still outperform the basic scheme. For $M=8$, when $L=10$ the random-phase scheme could achieve around $0.4 \mathrm{~dB}$ and $0.2 \mathrm{~dB}$ gains in the low- and high-SNR regime, respectively; when $L=100$ the random-phase scheme could achieve around $0.6 \mathrm{~dB}$ and 0.3 $\mathrm{dB}$ gains in the low- and high-SNR regimes, respectively.

The overall implications of our analytical and simulations results are as follows. The equal-SNR scheme, with the target of achieving perfect fairness among the links, is also a good approximation to the maxmin problem when the relay noise is small. Given a symmetric switch matrix that realizes pairwise transmissions, we could use the counter-phase algorithm or the random-phase algorithm to identify a suitable gain vector. The gap between the two algorithms is small, but the counter-phase algorithm has faster execution time. Given an asymmetric switch matrix that realizes non-pairwise transmissions, we could use the random-phase algorithm to identify a suitable gain vector.

\section{CONCLUSION}

We have proposed a framework for wireless MIMO switching to facilitate communications among multiple wireless stations. We focused on designing the precoders of our MIMO switching system. Our general conclusion is that we can use the solution of the equal-SNR problem to approximate that of the maxmin problem, which is NP-hard. In addition, we discover an interesting result for pairwise switch matrices i.e., the switch matrices are even-dimensional and symmetric. The computation cost of finding the optimal precoders could be high in general. However, when the switch matrix is evendimensional and symmetric, the computation of the precoder can be much simplified with a "counter-phase" approach. This approach, however, can not be applied in the case of the nonpairwise transmissions.

We propose a random-phase algorithm that are suitable for both pairwise and non-pairwise transmissions. This algorithm has good performance for both cases, with a modest increase in complexity.

There are many future directions going forward. For example, the precoders used in our simulation studies could be further optimized. Physical-layer network coding could be considered to improve throughput performance [10]. In addition, this paper has only considered switch matrices that realize permutations in which each station transmits to one and only one other station and receives from one and only one other station; it will be interesting to explore switch matrices that realize connectivities among stations that are not strictly permutations. Finally, future work could also explore the case where the number of antenna at the relay is not exactly $N$.

\section{REFERENCES}

[1] D. Gunduz, A. Yener, A. Goldsmith, and H. V. Poor, "The multi-way relay channel," in Proc. IEEE Int'l Symp. Inform. Theory, 2009, pp. 339-343.

[2] Y. E. Sagduyu, D. Guo, and R. A. Berry, "Throughput optimal control for relay-assisted wireless broadcast with network coding," in IEEE International Workshop on Wireless Network Coding (WiNC), San Francisco, CA, USA, Jun. 2008.

[3] T. Cui, T. Ho, and J. Kliewer, "Space-time communication protocols for N-way relay networks," in IEEE Global Telecommunications Conference, 2008, pp. 1-5.

[4] F. Gao, T. Cui, B. Jiang, and X. Gao, "On communication protocol and beamforming design for amplify-and-forward N-way relay networks," in 3rd IEEE International Workshop on Computational Advances in MultiSensor Adaptive Processing (CAMSAP), 2009.

[5] Y. Mohasseb, H. Ghozlan, G. Kramer, and H. El Gamal, "The MIMO wireless switch: Relaying can increase the multiplexing gain," in Proc. IEEE Int'l Symp. Inform. Theory, 2009, pp. 1448-1552.

[6] F. Wang and S. C. Liew, "Wireless MIMO switching," submitted to IEEE Trans. Wireless Commun., Apr. 2011. [Online]. Available: http://arxiv.org/abs/1104.4035v1

[7] Wikipedia, "Quartic function - Wikipedia, the free encyclopedia," 2011. [Online]. Available: http://en.wikipedia.org/wiki/Quartic_equation

[8] N. D. Sidiropoulos, T. N. Davidson, and Z.-Q. Luo, "Transmit beamforming for physical-layer multicasting," IEEE Trans. Signal Process., vol. 54, no. 6, pp. 2239-2251, 2006.

[9] A. U. T. Amah and A. Klein, "Non-regenerative multi-way relaying with linear beamforming," in Proc. 20th IEEE International Symposium on Personal, Indoor and Mobile Radio Communications Symposium, 2009.

[10] S. Zhang, S. C. Liew, and P. P. Lam, "Hot topic: physical-layer network coding," in in Proc. of ACM Mobicom, 2006. 